\title{
QUALIDADE DA CARNE SUÍNA APÓS DOIS TEMPOS DE DESCANSO NO FRIGORÍFICO
}

\author{
(Pork meat quality after two times of lairage in slaugther)
}

\author{
KÖHLER, R.G.; FREITAS, R.J.S. \\ Programa de Pós-graduação em Tecnologia de Alimentos - UFPR - C. Postal 19011, CEP 81531-990,
} Curitiba, PR. E-mail: robson.kohler@bol.com.br.

RESUMO - Existem vários fatores estressantes que podem afetar a qualidade da carne e também há falta de informação sobre a quantidade de horas mínimas e máximas ideais de descanso no frigorífico para a reposição do esgotamento físico dos animais após o transporte. O presente trabalho teve como objetivo estudar o efeito da redução do tempo de permanência na pocilga do frigorífico sobre a qualidade da carne suína, avaliando-se as seguintes características: $\mathrm{pH}$; capacidade de retenção de água (WHC). A redução de descanso de 9 horas para 3 horas de descanso no frigorífico não alteraram de modo significativo a qualidade da carne suína nas variáveis $\mathrm{pH} 45$ minutos, $\mathrm{pH} 24$ horas pós abate e na capacidade de retenção de água (WHC) pelos métodos Drip Loss e papel de filtro prensado de GRAU e HAMM, mas com a redução do tempo de descanso no frigorífico, pôdese obter outros ganhos (redução do estresse, escoriações de pele, hematomas, fraturas e outros) e em qualidade da carne.

Palavras-chave: Qualidade de carne; Descanso; pH.; WHC; Suíno.

ABSTRACT - There are several stressing factors that can affect the meat quality, and also a lack of information on the minimum and maximum ideal lairage time in the slaugther for the replacement of animal physical exhaustion after transportation. The reduction of 9 to 3 hours of lairage in the preslaugther did not significantly alter the pork meat quality in the variables $\mathrm{pH} 45 \mathrm{~min}, \mathrm{pH} 24 \mathrm{~h}$ postslaugther, water-holding capacity (WHC) for the methods Drip Loss and filter paper press of GRAU \& HAMM. However, with the reduction lairage time in the slaugther, other gains could be obtained (reduction of stress, skin excoriations, hematomas, fractures and others) as well as increase in meat quality.

Key-words: Meat Quality; Lairage; pH.; WHC; Swine.

\section{Introdução}

O investimento na produção de suínos (genética, nutrição, sanidade e sistemas de produção) foi crescente nos últimos anos e teve como objetivo a obtenção de um animal competitivo. No entanto, o novo modelo de animal, trouxe desafios, principalmente nos aspectos de manejo pré-abate (SILVEIRA, 2001).

Uma considerável e significativa variação na qualidade da carne do suíno é verificada nos frigoríficos brasileiros, europeus e norteamericanos (CULAU et al., 1994), onde as carnes destinadas à industrialização poderão apresentar diversos problemas, entre os quais destacam-se as carnes Pálida, Mole e Exsudativa (PSE) e
Escura, Dura e Seca (DFD) (TERRA, 1998).

Existem vários outros fatores que podem influenciar à incidência da carnes PSE, além dos fatores genéticos: o tempo de transporte dos animais da granja para o frigorífico (ANDRADE et al., 1992), o manejo utilizado no pré-abate do frigorífico (SOUZA et al., 1998), o tempo de descanso dos animais antes do abate (ANDRADE et al., 1992), os métodos de atordoamento (VELARDE et al., 2000), o intervalo de tempo entre sangria e a entrada da carcaça na câmara de resfriamento (BRESSAN et al., 1992).

A falta de preocupação para a qualidade da carne e a inabilidade da indústria para medi-la e classificá-la objetivamente são a razão de um 
aumento da baixa da qualidade da carne suína. São vários os problemas associados com a avaliação da qualidade do músculo no post mortem. As variações de cor ou capacidade de retenção de água (WHC) no músculo suíno não são verificadas no abate ou na linha de corte, porque ainda não existem métodos rápidos, econômicos e com precisão para detectá-las, especialmente para WHC (JOO et al., 2000b).

Tendo em vista que as alterações musculares podem se manifestar dentro da primeira hora após o abate, vários parâmetros têm sido avaliados aos 45 minutos após o abate para predizer a qualidade final da carne entre 20 e 24 horas após o abate (WARRISS e BROWN, 1987). As variáveis que melhor definem a qualidade da carne suína em ordem de importância são o pH nas 24 horas, o pH aos 45 minutos, a medida de fibra óptica e a medida de perda de líquido por gotejamento. $O$ intervalo de variação dos parâmetros de $\mathrm{pH}$, WHC, temperatura, cor, textura são analisados e associados de modo a qualificar a carne suína (OURIQUE e NICOLAIEWSKY, 1990)

Há evidência suficiente para acreditar que os fatores mais importantes para a utilização da classificação de qualidade da carne suína sejam o $\mathrm{pH}$, cor e WHC. Assim, $\mathrm{pH}_{24} \mathrm{~h}$, cor - L * e Drip Loss são escolhidos como as principais variáveis usadas para o critério de seleção e classificação da qualidade da carne suína (JOO et al., 2000 a).

O trabalho teve como objetivo estudar o efeito da redução do tempo de permanência dos animais na pocilga do frigorífico sobre a qualidade da carne suína avaliando-se o $\mathrm{pH}$ aos 45 minutos, nas 24 horas após abate e WHC, utilizando os métodos Drip Loss e papel de filtro prensado de GRAU e HAMM.

\section{Material e Métodos}

Foram utilizados 320 animais para a realização do teste de descanso de 3 horas e 320 animais para o teste de descanso 9 horas. Os animais foram mantidos na propriedade em jejum e dieta hídrica por 12 horas e posteriormente foram transportados por 2:50 horas, em caminhões, até o frigorífico. Neste, os animais ficaram em baias, separadas, conforme o teste a ser realizado, por 3 horas e 9 horas de descanso e dieta hídrica, respectivamente. Após o abate, já na câmara fria, coletou-se os valores de $\mathrm{pH}$ aos 45 minutos e nas 24 horas no $M$. semimembranosus, seguindo a metodologia citada por BAAS (2000) e coletou-se amostras do $M$. semimembranosus para avaliação Drip Loss (perda por gotejamento), seguindo a técnica desenvolvida por HONIKEL (1988), e para a técnica de papel de filtro prensado desenvolvida por GRAU e HAMM (1953). O teste estatístico utilizado foi o teste $F$, considerando-se no nível de $5 \%$.

\section{Resultados e Discussão}

Resultados do $\mathrm{pH}$ aos 45 minutos e nas 24 horas pós abate: As médias do $\mathrm{pH}$ aos 45 minutos e nas 24 horas após abate, utilizando os dados das amostras do $M$. semimembranosus e foram relacionadas conforme o tratamento de 3 e 9 horas e estão dispostas nas FIGURAS 1 e 2.

A FIGURA 1 mostra as médias obtidas para os tratamentos de 3 e 9 horas e que são semelhantes, não havendo diferença estatística no nível de $5 \%$ entre os tratamentos em relação ao $\mathrm{pH}$ aos 45 minutos após o abate.

\section{FIGURA 1 - MÉDIAS DE pH AOS 45 MINUTOS DOS TRATAMENTOS 3 E 9 HORAS DE DESCANSO APLICADO AOS SUÍNOS, 2001. $(n=640)$.}

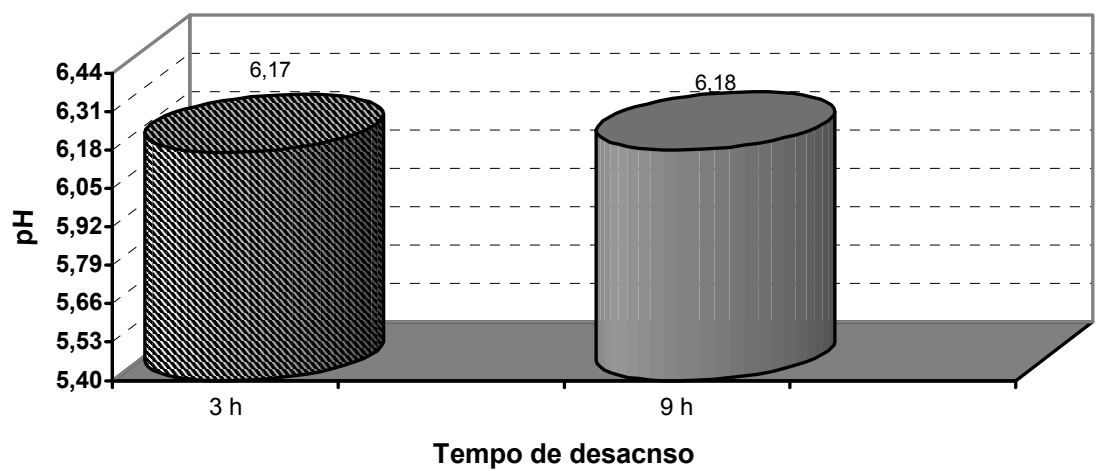


Trabalhos realizados por DE SMET et al. (1996) e OWEN et al. (2000) com tempos de descanso variados, demonstraram um $\mathrm{pH}$ de 6,16 aos 45 minutos para o tempo $2-3$ horas, $\mathrm{pH}$ muito semelhante ao deste trabalho $(6,17)$ para o tempo de 3 horas de descanso na pocilga do frigorífico.

A FIGURA 2 evidencia que as médias obtidas para os tratamentos são muito similares e que não há diferença estatística no nível de $5 \%$ entre os tratamentos em relação ao $\mathrm{pH}$ nas 24 horas após o abate.

\section{FIGURA 2 - MÉDIAS DE pH NAS 24 HORAS DOS TRATAMENTOS 3 E 9 HORAS DE DESCANSO APLICADO AOS SUÍNOS, 2001. $(n=640)$.}

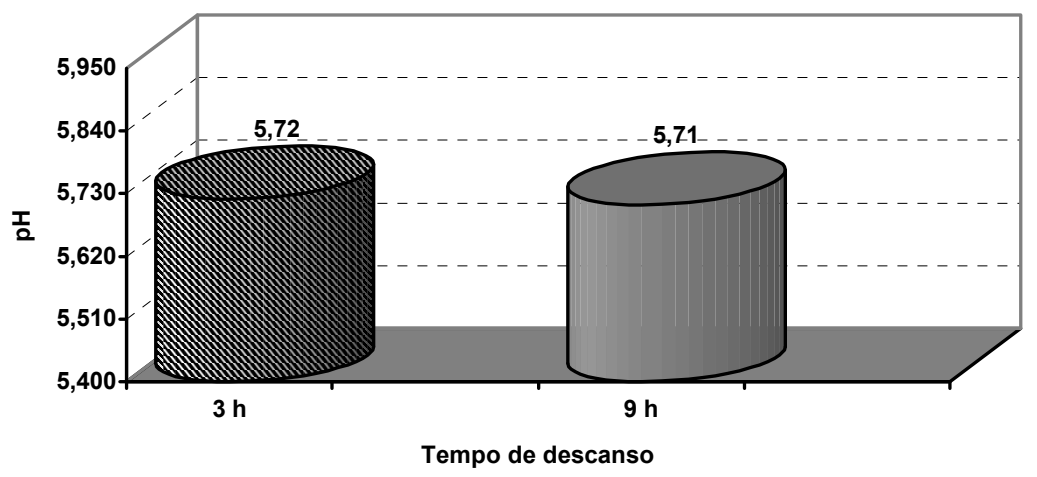

DE SMET et al. (1996) testando diferentes tempos, registraram valores de $\mathrm{pH}$ nas 24 horas após o abate, nos tempos <1 e 4-5 horas de descanso, abaixo do obtido neste trabalho $(5,69$ e 5,68), mas no teste de descanso de $2-3$ horas obteve um $\mathrm{pH}$ semelhante $(5,71)$, ao deste trabalho (com descanso de 9 horas) e muito similar ao de descanso de 3 horas.

SANTOS et al. (1997) observando a diferença entre dois tempos de descanso na pocilga do frigorífico (30 minutos e 2-3 horas), obtiveram $\mathrm{pH}$ nas 24 horas muito abaixo do obtido nesta pesquisa $(5,59$ e 5,65$)$, devido terem trabalhado com várias temperaturas e umidade relativas diferentes.

OWEN et al. (2000) encontraram resultados de $\mathrm{pH}$, muito abaixo para todos os testes de 0 , 1,2 e 3 horas de descanso em relação ao desta pesquisa. A possível causa da grande diferença, reside no local escolhido para se fazer a medição ( $M$. semimembranosus em nosso trabalho) sendo que os autores escolheram o $M$. longissimus dorsis.

Nossos resultados são corroborados por GISPERT et al. (2000), os quais igualmente não obtiveram diferença no $\mathrm{pH}$ às 24 horas nos mesmos tempos de 3 e 9 horas de descanso.

Resultados da WHC pelos métodos Drip Loss e papel de filtro prensado de GRAU e HAMM: As médias do Drip Loss utilizando amostras do $M$. semimembranosus foram relacionadas conforme o tratamento e estão dispostas na FIGURA 3.

Com relação ao teste de WHC, (FIGURA 3) pode-se evidenciar que o tratamento onde os animais que permaneceram 6 horas de descanso a mais, houve $0,5 \%$ a mais de perda por gotejamento no teste Drip Loss, havendo assim pequena diferença e melhoria na qualidade da carne nos animais que ficaram 3 horas de descanso. Estatisticamente no nível de $5 \%$, não houve diferença entre os tratamentos de 3 e 9 horas, para Drip Loss. 
FIGURA 3 - MÉDIAS DE DRIP LOSS DOS TRATAMENTOS 3 E 9 HORAS TEMPO DE DESCANSO APLICADO AOS SUÍNOS, 2001. $(n=640)$.

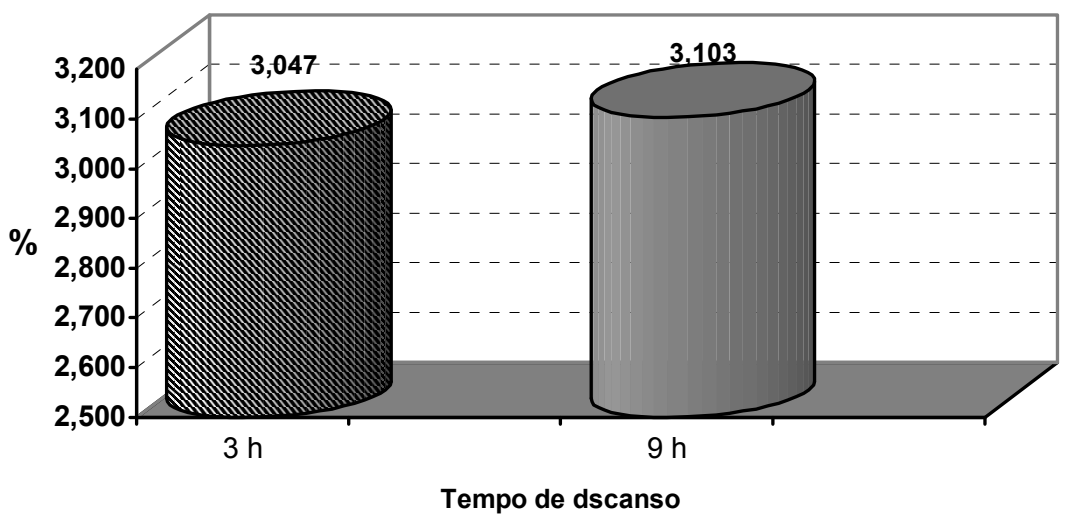

DE SMET et al. (1996), fazendo teste com diferentes tempos de descanso $(<1,2-3$ e 4-5 horas), e SOUZA et al. (1998), utilizando o mesmo tempo de descanso ( 15 horas) para dois grupos de animais testes, sendo um grupo de animais com o mínimo de estresse no manejo e o outro grupo de animais com o manejo estressante (utilizando choque), obtiveram resultados acima dos encontrados neste trabalho, observando-se significativas perdas por gotejamento que variaram de $4,5 \%$ a $10,4 \%$.

No resultado as médias do papel de filtro prensado de GRAU e HAMM (1953), utilizando amostras do $M$. semimembranosus foram relacionadas conforme o tratamento e estão dispostas na FIGURA 4.

\section{FIGURA 4 - MÉDIAS DO PAPEL DE FILTRO PRENSADO DE GRAU E HAMM DOS TRATAMENTOS 3 E 9} HORAS DE DESCANDO APLICADO AOS SUÍNOS, 2001. ( $\mathrm{N}=640)$.

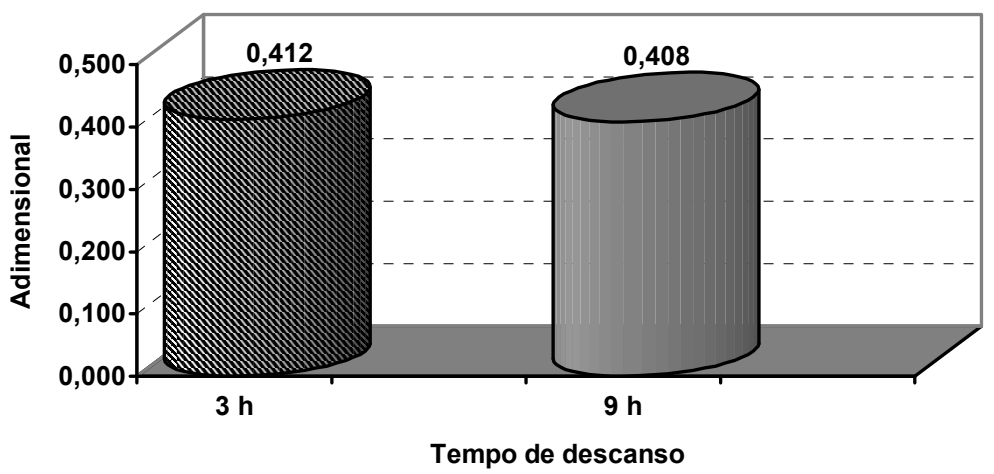

Ao se analisar a FIGURA 4, observa-se que não há diferença estatística no nível de $5 \%$ entre os tratamentos 3 e 9 horas, considerandose o método de papel de filtro prensado de GRAU e HAMM.

Em pesquisa realizada por SVOBODA (1998) executou-se testes de congelamento de carnes suínas desossadas imediatamente após o abate dos animais. As carcaças foram desossadas a quente, ocorrendo influência de diversas variáveis relacionados à qualidade da carne suína, em animais com muita facilidade de se estressarem. Segundo esse autor a característica do WHC pelo método do papel de filtro prensado de GRAU e HAMM (1953), a desossa convencional (desossa das 
carcaças um dia após ao abate) de animais PSE, resultou em 0,352 (leitura no planímetro), melhores que os dos animais padrões, não PSE ou normais, os quais resultaram em 0,437 . Nossos achados situaram-se entre 0,412 e 0,408 da medida do planímetro.

No confronto entre nossos resultados $(0,412$ e 0,408$)$ e o de SVOBODA (1998) $(0,437$, resultado esses dos animais não PSE), observa-se pequena diferença entre os dados dos dois testes (tempos de descanso e abate em confronto com o teste da desossa convencional de animais não PSE). Tal diferença possivelmente possa ser atribuída à leitura das áreas (carne e líquido), em função de que o planímetro é extremamente sensível ao ser manuseado.

\section{Conclusões}

Nas condições da realização do presente trabalho, os resultados obtidos permitem concluir que o efeito da redução do tempo de permanência na pocilga do frigorífico sobre a qualidade da carne suína apresentou:

- Nos tratamentos de 3 e 9 horas de descanso no frigorífico, não houve alteração de modo significativo sobre a qualidade da carne suína, quando avaliados os parâmetros: de $\mathrm{pH}$, medido aos 45 minutos e nas 24 horas após abate; da capacidade de retenção de água (WHC), pelos métodos de Drip Loss e do papel de filtro prensado de GRAU e HAMM e pôde-se obter outros ganhos (redução do estresse, escoriações de pele, hematomas, fraturas e outros). Com base nesses resultados sugere-se a redução do período de descanso dos animais nas pocilgas dos frigoríficos, visto que os animais que permaneceram 3 horas na pocilga do frigorífico demonstraram valores semelhantes àqueles mantidos por 9 horas. Finalmente sugere-se que a legislação em vigor (RIISPOA de 1962) seja modificada, especificamente para a espécie suína, no seu Art. $110, \S 1^{\circ}$ do Capítulo I: Inspeção "Antemortem".

\section{REFERÊNCIAS}

ANDRADE JÚNIOR, R.; NICOLAIEWSKY, S.; OURIQUE, J.M.R.; CULAU, P.O.V.; BRESSAN, M.C. Análise de alguns fatores determinantes da qualidade da carne suína. I. Efeito da distância GranjaFrigorífico, tempo de descanso, sexo e peso-vivo. In: CONGRESSO BRASILEIRO DE MEDICINA VETERINÁRIA, 22., 1992, Curitiba. Anais... Curitiba, 1992. p.216.

BAAS, T. J. Pork Composition \& Quality. Assessment procedures pork the other white meat. University of Missouri, National Pork Producers Council, lowa, 2000. 41p.

BRASIL. Ministério da Agricultura. Regulamento da inspeção industrial e sanitária de produtos de origem animal - RISPOA. Brasília: MA, 1962. Disponível em:< http://www. agricultura.gov.br/sda/ dipoa/riispoa.html\#títulovii >. Acesso em: $16 \mathrm{dez}$. 2000.

BRESSAN, M.C.; CULAU, P.O.V.; OURIQUE, J.M.R.; NICOLAIEWSKY, S. Effect of between bleeding and the entry of carcasses in chilling chamber and chilling rates on pork quality. In: INTERNATIONAL CONGRESS of MEAT SCIENCE and TECHNOLOGY, 38., 1992. Clermont-Ferrand. Proceeding. Clermont-Ferrand, France, 1992. v.2, p.165-168.

CULAU, P.O.V.; OURIQUE, J.M.R.; NICOLAIEWSKY,.S.; BRESSAN, M.C. Incidence of PSE in commercial pig carcasses in Rio Grande do Sul state, Brazil. Boletim Técnico da Associação Sul Brasileira das Indústrias de Produtores Suínos, Porto Alegre, 1994.

DE SMET, M.S.; PAUWELS, H.; DE BIE, S.; DEMEYER, I.D.; CALLEWIER, J.; EECKHOUT, W. Effect of halothane genotype, breed, feed withdrawal, and lairage on pork quality of Belgian slaughter pigs. Journal of Animal Science, Savoy, n.74, p.185463, 1996.

GISPERT, M.; FAUCITANO, L.; GUARDIA, M.D.; OLIVER, M.A.; SIGGENS, K.; HARVEY, K.; DIESTRE, A. A survey on pre-slaughter conditions, halothane gene frequency, and carcass and meat quality in five Spanish pig commercial abattoirs. Meat Science, Barking, v.55, p.97-106, 2000.

GRAU, R.; HAMM, R. Eine einfache Methode zur Bestimmung der Wasserbindung im Fleish. Fleischwirtschaft, Frankfurt, v.4, p.295-297, 1953. HONIKEL, K.O. Capacidad de fijación de agua de la carne. Fleischwirtschaft (ed. espanhola), Frankfurt, n.1, p.12, 1988. 
JOO, T.S.; KAUFFMAN, G.R.; WARNER, D.R.; BORGGAARD, C.; STEVENSON-BARRY, M.J.; LEE, S.; PARK, B.G.; KIM, C.B. Objectively predicting ultimate quality of post-rigor pork musculature: I. Initial comparison of techniques. Asian - Australasian Journal of Animal Sciences, Suwon, v.13, n.1, p.68-76, 2000a.

JOO, T.S.; KAUFFMAN, G.R.; WARNER, D.R.; BORGGAARD, C.; STEVENSON-BARRY, M.J.; LEE, S.; PARK, B.G.; KIM, C.B. Objectively predicting ultimate quality of post-rigor pork musculature: II. Pratical classification method on the cutting-line. Asian - Australasian Jornal of Animal Sciences, Suwon, v.13, n.1, p.77-85, 2000b.

OURIQUE, M.J.; NICOLAIEWSKY, S. Características físico-químicas e organolépticas e suas relações na avaliação de qualidade da carne suína. Revista Sociedade Brasileira de Zootecnia, Viçosa, v.19, n.2, p.118-125, 1990.

OWEN, L.B.; MONTGOMERY, L.J.; RAMSEY, B.C.; MILLER, M.F. Preslaughter resting and hot-fat trimming effcts on the incidence of pale, soft and exudative (PSE) pork and ham processing characteristics. Meat Science, Barking, v.54, p.221-229, 2000.

SANTOS, C.; ALMEIDA, J.M.; MATIAS, E.C.; FRAQUEZA, M.J.; ROSEIRO, C.; SARDINA, L. Influence of lairage environmental conditions and resting time on meat quality in pigs. Meat Science, Barking, v.45, p.253-262, 1997.

Recebido para publicação: 18/11/2004 Aprovado:
SILVEIRA, F.T.E. Carne sem estresse. Suinocultura Industrial, Porto Feliz, fev./mar., p.31-32, 2001.

SOUZA, N.D.; WARNER, F. R.; DUNSHEA, R.F.; LEURY, B.J. Effect of on- farm and pre-slaughter handling of pigs on meat quality. Australian Journal Agricultural Research, Collingwood, n.49, p.1021-25, 1998.

SVOBODA, K.W. Estudo da carne suína PSE em relação ao resfriamento e textura. Campinas: 1998.133 p. Dissertação (Mestrado em Tecnologia de Alimentos) - Programa de PósGraduação em Tecnologia de Alimentos, Universidade Estadual de Campinas.

TERRA, N.N. Apontamentos de tecnologia de carnes. São Leopoldo: Ed. UNISINOS, 1998. $216 \mathrm{p}$.

VELARDE, A.; GISPERT, M.; FAUCITANO, L.; MANTECA, X.; DIESTRE, A. The efect of stunning method on the incidence of PSE meat and haemorrhages in pork carcasses. Meat Science, Barking, v.55, p.309-314, 2000.

WARRISS, P.D.; BROWN, S.N. The relationships between initial $\mathrm{pH}$, reflectance and exudation in pig muscle. Meat Science, Barking, v.20, p.65$74,1987$. 